Ophthalmologe 2015 · 112:710-711

DOI 10.1007/s00347-015-0124-5

Online publiziert: 26. August 2015

(c) Springer-Verlag Berlin Heidelberg 2015

\section{K.U. Bartz-Schmidt · M. Ueffing}

Universitäts-Augenklinik und Forschungsinstitut für Augenheilkunde, Department

für Augenheilkunde, Universitätsklinikum Tübingen, Tübingen, Deutschland

\title{
Behandlung heredodegenerativer Netzhauterkrankungen heute und in Zukunft
}

Bis heute sind über 200 heredodegenerative Netzhauterkrankungen genetisch entschlüsselt. Bisher galten diese Erkrankungen, von denen alleine in Deutschland mehr als 40.000 Menschen betroffen sind, als unheilbar. Gekeler et al. berichten nun in diesem Heft, wie Entwicklungen in den 1990er-Jahren dazu geführt haben, dass inzwischen 2 zugelassene Netzhautsehprothesen verfügbar sind. Beide ermöglichen den Patienten mit einem genetisch bedingten Funktionsverlust auf Ebene der Photorezeptoren und des retinalen Pigmentepithels, aber intaktem nachgeschalteten neuroretinalen Netzwerk genunabhängig die Wiedererlangung einer basalen Sehfunktion. Durch Umwandlung des Lichtreizes in eine auf die Netzhaut projizierte elektrische Stimulation konnten für einzelne Patienten in einem kleinen zentralen Gesichtsfeldausschnitt Sehschärfen von bis zu umgerechnet 20/200 erzielt werden. Während das subretinale Implantat die bipolaren Zellen direkt stimuliert, wird die Umgebungswahrnehmung im Auge beim epiretinalen Implantat durch Übertragung und elektrische Kodierung eines Videostreams auf die Ganglienzellen übertragen. Dieser prothetische Ersatz degenerierter Zellen ist allerdings nur für solche Patienten geeignet, die in ihrer Kindheit bereits das Sehen gelernt haben, da Netzhautprothesen nur über eine Ankopplung an das visuelle System funktionieren können. Diese Einschränkung gilt für Netzhautprothesen genauso wie für die Gentherapie und Zellersatztherapie.

Während Sehprothesen jedoch nicht in der Lage sind, die Ursachen der Er- krankung auszuräumen, versuchen Genund Stammzelltherapie, das Krankheitsgeschehen kurativ zu beeinflussen. Damit besteht prinzipiell die Möglichkeit, Letztere nicht erst nach einer eingetretenen Erblindung zum Einsatz zu bringen, sondern schon zur Vermeidung einer funktionellen Verschlechterung des Sehens. Nach derzeitigem Stand sind, wie Bellingrath und Fischer in diesem Heft darlegen, die Möglichkeiten der Gentherapie gerade bei den monogenetisch determinierten Erkrankungen als aussichtreicher anzusehen als die Zellersatztherapie mittels Transplantation induzierter pluripotenter Stammzellen (iPS).

\section{》) Gen-und \\ Stammzelltherapie versuchen, das Krankheitsgeschehen kurativ zu beeinflussen}

iPS-Zellen stammen, wie Balmer, Stanzel und Fischer in einem dritten Beitrag in diesem Heft zeigen, aus dem Organismus des Erkrankten und tragen damit auch dessen Gendefekt. Eine Alternative wäre der Einsatz embryonaler Stammzellen oder allogener Stammzellen aus gesunden Spendern, wie sie bei der Knochenmarktransplantation erfolgreich etabliert ist. Der besondere Charme der Zellersatztherapien liegt darin, dass ein wesentlich breiteres Indikationsspektrum, z. B. das der altersabhängigen Makuladegeneration, auch im Fokus der Entwicklungen steht.

Am Horizont erscheinen aber bereits noch faszinierendere Behandlungsverfah- ren. So haben verschiedene Gruppen, allen voran die Arbeitsgruppe von Rudolf Jaenisch [1], Verfahren zu molekularen Reparaturstrategien durch gezielte Veränderung oder Reparatur des Genoms entwickelt. Mittels CRISPR/CAS-vermitteltem Genome-Engineering konnte gezeigt werden, wie im Zellkern an der DNA gezielt DNA-Abschnitte erkannt, geschnitten und ausgetauscht werden können. Damit ist vorstellbar, erkrankungsassoziierte Mutationen im Patienten gezielt durch Sequenzkorrektur zu beseitigen. Vorausgesetzt, die von einem Gendefekt betroffenen Zellen regenerieren sich, könnte somit die Erkrankung durch Genkorrektur geheilt werden. Allerdings werfen die Möglichkeiten dieser neuen Technologien neue, weit tiefer greifende ethische Fragen auf als diejenigen, welche zur derzeitigen Gesetzgebung im Bereich Embryonenschutz und Stammzellnutzung in Deutschland geführt haben. Wir haben jetzt einen Punkt erreicht, wo Forschung nicht nur unser Feld, die Augenheilkunde, verändert, sondern auch die Gesellschaft, in der wir leben. Molekular- und Zellbiologie haben damit gesellschaftlich umwälzende Entwicklungen angestoßen, deren Weg ethisch begleitet werden muss.

Die zum Leitthema ausgewählten Beiträge zeigen nicht nur Perspektiven einer neuen molekularen und zellbasierten Medizin auf, sie verdeutlichen auch, wie innerhalb weniger Jahre aus internationalen und vor allem interdisziplinären Forschungsanstrengungen enorme Fortschritte für unser Feld erzielt wurden. Daran teilzuhaben und mitzugestalten ist überwältigend und spannend zugleich. 


\section{In eigener Sache}

\section{$1 / H-10$}

K.U. Bartz-Schmidt<smiles>CCCCCCCCCCCCCCCCCCCC</smiles>

\section{Ueffing}

\section{Korrespondenzadresse}

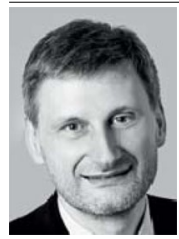

Prof. Dr. K.U. Bartz-Schmidt

Universitäts-Augenklinik und Forschungsinstitut für Augenheilkunde, Department für Augenheilkunde Universitätsklinikum Tübingen Schleichstr. 12

72076 Tübingen

u.bartz-schmidt@uni-tuebingen.de

Interessenkonflikt. K.U. Bartz-Schmidt und M. Ueffing geben an, dass kein Interessenkonflikt besteht.

\section{Literatur}

1. Wang $\mathrm{H}$, Yang $\mathrm{H}$, Shivalila CS, Dawlaty MM, Cheng AW, Zhang F, Jaenisch R (2013) One-step generation of mice carrying mutations in multiple genes by CRISPR/Cas-mediated genome engineering. Cell 153(4):910-918

\section{Immer aktuell - Dank Online-First}

\section{Sichern Sie sich mit Online-First Beiträgen einen Wissensvorsprung!}

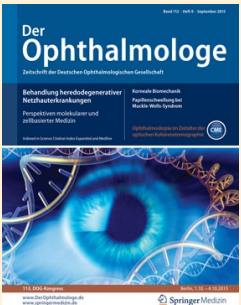

Mit der Online-First-Veröffentlichung aller Beiträge aus Der Ophthalmologe bieten wir Ihnen die Möglichkeit, bereits wenige Wochen nach Einreichung in den neuesten Originalien und Kasuistiken zu recherchieren.

Die Beiträge sind sofort auffindbar und durch den DOI (Digital Object Identifier) ist die Zitierfähigkeit gewährleistet.

Folgende Beiträge wurden im August 2015 Online-First veröffentlicht:

- Verdickung oder Hypoplasie der Fovea.

Nessmann A, Schramm C, Gelisken F.

DOI 10.1007/s00347-015-0113-8

- Langzeitergebnisse zur autologen Transplantation von ex vivo kultiviertem Limbusepithel bei limbaler Stammzellinsuffizienz.

Scholz S L, Thomasen H, Hestermann K, Dekowski D, Steuhl K-P, Meller D.

DOI 10.1007/s00347-015-0110-y

- Rezidivierende Keratokonjunktivitis.

Höck A, Löffler KU.

DOI 10.1007/s00347-015-0114-7

Sie finden alle Online-First-Beiträge für Der Ophthalmologe unter http://link.springer.com/journal/347/onlineFirst

Wir wünschen Ihnen eine anregende Lektüre.

Ihre Redaktion Der Ophthalmologe 